\title{
NGF Regulates the Expression of Axonal LINGO-1 to Inhibit Oligodendrocyte Differentiation and Myelination
}

\author{
Xinhua Lee, ${ }^{1}$ Zhongshu Yang, ${ }^{1}$ Zhaohui Shao, ${ }^{1}$ Sheila S. Rosenberg, ${ }^{2}$ Melissa Levesque, ${ }^{1}$ R. Blake Pepinsky, ${ }^{1}$ \\ Mengsheng Qiu, ${ }^{3}$ Robert H. Miller, ${ }^{4}$ Jonah R. Chan, ${ }^{2}$ and Sha Mi ${ }^{1}$ \\ ${ }^{1}$ Department of Discovery Biology, Biogen Idec, Cambridge, Massachusetts 02142, ${ }^{2}$ Department of Cell and Neurobiology, Zilkha Neurogenetic Institute, \\ University of Southern California, Keck School of Medicine, Los Angeles, California 90033, ${ }^{3}$ Department of Anatomy and Neurobiology, University of \\ Louisville, Louisville, Kentucky 40202, and ${ }^{4}$ Department of Neurosciences, Case School of Medicine, Cleveland, Ohio 44106
}

\begin{abstract}
Neurons and glia share a mutual dependence in establishing a functional relationship, and none is more evident than the process by which axons control myelination. Here, we identify LRR and Ig domain-containing, Nogo receptor-interacting protein (LINGO-1) as a potent axonal inhibitor of oligodendrocyte differentiation and myelination that is regulated by nerve growth factor and its cognate receptor TrkA in a dose-dependent manner. Whereas LINGO-1 expressed by oligodendrocyte progenitor cells was previously identified as an inhibitor of differentiation, we demonstrate that axonal expression of LINGO-1 inhibits differentiation with equal potency. Disruption of LINGO-1 on either cell type is sufficient to overcome the inhibitory action and promote differentiation and myelination, independent of axon diameter. Furthermore, these results were recapitulated in transgenic mice overexpressing the full length LING0-1 under the neuronal promoter synapsin. Myelination was greatly inhibited in the presence of enforced axonal LINGO-1. The implications of these results relate specifically to the development of potential therapeutics targeting extrinsic growth factors that may regulate the axonal expression of modulators of oligodendrocyte development.
\end{abstract}

Key words: oligodendrocyte; myelination; LINGO-1; neurotrophin; differentation; dorsal root ganglion

\section{Introduction}

The formation of the myelin sheath is an exquisite and dynamic example of cell-cell interaction that involves the myelin-forming cell and the neuronal axon. It is generally believed that during development axons will control the proliferation, survival, and differentiation of the oligodendrocyte progenitor cell (OPC). Although the axonal factors that induce differentiation and myelination are still in question, it is well accepted that axons also control whether they will become myelinated by expressing appropriate signals to either promote or inhibit this process (Colello and Pott, 1997). Although some axons are myelinated and others remain unmyelinated, what are the factors that determine the expression of these signals along axons, what are these signals, and how are these signals regulated throughout development?

Myelination of axons is dictated, in large part, by axonal signals that control the development of myelinating glia, and it has long been thought that axon diameter is the key determining factor for the initiation of myelination. This notion has been revisited in recent years and numerous studies concerning the

Received Sept. 22, 2006; revised Nov. 27, 2006; accepted Nov. 29, 2006.

This work was supported in part by grants from the National Multiple Sclerosis Society Career Transition Award and the Donald E. and Delia B. Baxter Foundation Award to J.R.C.

Correspondence should be addressed to either of the following: Dr. Jonah R. Chan, Zilkha Neurogenetic Institute, Keck School of Medicine at University of Southern California, Department of Cell and Neurobiology, 1501 San Pablo Street ZNI 421, Los Angeles, CA 90033, E-mail:jonah.chan@usc.edu; or Dr. Sha Mi, Biogen Idec, Discovery Biology, 14 Cambridge Center, Cambridge, MA 02142, E-mail: sha.mi@biogenidec.com.

D01:10.1523/JNEUROSCI.4175-06.2007

Copyright $\odot 2007$ Society for Neuroscience $\quad$ 0270-6474/07/270220-06\$15.00/0 axonal regulation of myelination have attempted to address this particular assumption. Specifically, the finding that axonal neuregulin-1 type III regulates the ensheathment and thickness of the myelin sheath formed in the peripheral nervous system (PNS) by Schwann cells (SCs) has stimulated additional studies addressing how this growth factor transduces its signal and is encoded into a physiological measure of axon size (Michailov et al., 2004; Taveggia et al., 2005). In addition to neuregulin-1 type III, two other growth factors have been reported to act similarly on sensory neurons and regulate the axonal signals that promote SC myelination. Glial cell line-derived neurotrophic factor (GDNF), a growth factor important for the development of a subset of nociceptive neurons, increases the proportion of those axons that are myelinated by SCs (Hoke et al., 2003). Similarly, nerve growth factor (NGF) acts on a different subset of nociceptive neurons expressing TrkA and is essential for myelination of these specific axons by SCs (Chan et al., 2004). It is quite possible that these two growth factors in the PNS share similar mechanisms promoting the expression of a common axonal signal (i.e., neuregulin-1 type III and initiate myelination).

Additionally, recent studies concerning NGF suggest that different axonal signals control central and peripheral myelination. This was observed by the unexpected and opposite effects of NGF on SC and oligodendrocyte myelination (Chan et al., 2004). To identify the axonal signals regulated by NGF that control oligodendrocyte myelination, we sought out axonal signals differentially regulated by NGF that would either promote or inhibit oligodendrocyte differentiation and myelination. Here, we iden- 
tify LRR and Ig domain-containing, Nogo receptor-interacting protein (LINGO-1), a molecule specific to the nervous system, as an axonal inhibitor of oligodendrocyte differentiation regulated by NGF. Whereas LINGO-1 expressed by oligodendrocytes was identified previously as an inhibitor of differentiation (Mi et al., 2005), we demonstrate that axonal expression of LINGO-1 inhibits with equal potency. Disruption of LINGO-1 on either cell type is sufficient to overcome the inhibitory action and promote differentiation and myelination, independent of axon diameter. These findings could provide important insights into our understanding of what constitutes a favorable axonal environment for oligodendrocyte maturation and novel therapeutic approaches for facilitating oligodendrocyte remyelination after demyelinating insult.

\section{Materials and Methods}

In situ hybridization. Rat dorsal root ganglia frozen sections were prepared and processed as described previously (Mi et al., 2000). Sections were probed with digoxigenin-labeled LINGO-1 antisense and sense RNA. Sections were stained using the Tyramide Signal Amplification (Amersham Biosciences, Piscataway, NJ) plus a fluorescence antidigoxigenin conjugated antibody kit (Perkin-Elmer, Wellesley, MA) following the manufacturer's instructions. For combined in situ hybridization and immunofluorescence analyses, cultures were first probed with the digoxigenin-labeled RNAs and then with anti-TrkA antibody (Santa Cruz Biotechnology, Santa Cruz, CA).

Immunostaining. To visualize antibody labeling in tissue sections or cell culture, samples were fixed in $4 \%$ paraformaldehyde and incubated with indicated antibodies using standard protocols. Briefly, tissue sections were incubated in primary antibodies overnight at $4^{\circ} \mathrm{C}$, washed thoroughly, incubated with the appropriate Alexa-labeled secondary antibody (Invitrogen, Eugene, OR) for $2 \mathrm{~h}$, and then mounted in VectaMount (Vector Laboratories, Burlingame, CA) and visualized by fluorescence microscopy. The anti-LINGO-1 antibody was generated from a human Fab phage display library (MorphoSys, Martinsried/Planegg, Germany). The anti-TrkA antibody was obtained from Santa Cruz Biotechnology.

Immunoprecipitation and Western blot analysis. Cultures were harvested and lysed in $1 \mathrm{ml}$ lysis buffer (50 mM HEPES, pH 7.5, $150 \mathrm{~mm}$ $\mathrm{NaCl}, 1.5 \mathrm{~mm} \mathrm{MgCl}$, 1 mm EGTA, 1\% Triton X-100 and 10\% glycerol). After centrifugation at $14,000 \times g$ for $15 \mathrm{~min}$, the supernatants were removed and incubated with Protein A/G Plus-Agarose beads (Santa Cruz Biotechnology) at $4^{\circ} \mathrm{C}$ for $1 \mathrm{~h}$, and then incubated at $4^{\circ} \mathrm{C}$ for $1 \mathrm{~h}$ with either affinity-purified rabbit anti-LINGO-1 antibody or mouse anti-Trk antibody (Santa Cruz Biotechnology). The beads were washed with lysis buffer, boiled in Laemmli sample buffer, subjected to SDS-PAGE, and analyzed by Western blot with a mouse anti-TrkA antibody (Santa Cruz Biotechnology) or anti-LINGO-1 antibody (Biogen Idec, Cambridge, MA). The TrkA and LINGO-1 antibodies were visualized using antimouse IgG-HRP and anti-rabbit IgG-HRP (Bio-Rad, Hercules, CA), respectively.

OPC culture. OPCs were isolated from female Long-Evans postnatal day 2 rats as described previously (Mi et al., 2005). Briefly, the forebrains were removed, diced into $1 \mathrm{~mm}$ fragments and incubated at $37^{\circ} \mathrm{C}$ for 15 min with trypsin and DNase. Dissociated cells were plated on poly-Llysine-coated tissue culture flasks and grown at $37^{\circ} \mathrm{C}$ for $10 \mathrm{~d}$ in DMEM medium with $20 \%$ fetal calf serum (Invitrogen). OPCs $\left(\mathrm{A} 2 \mathrm{~B} 5^{+}\right.$) were collected by shaking the flask overnight at $200 \mathrm{rpm}$ at $37^{\circ} \mathrm{C}$, resulting in $95 \%$ purity. Cultures were maintained in high-glucose DMEM medium containing FGF/PDGF (10 ng/ml; Peprotech, Rocky Hill, NJ) for 1 week.

$D R G$ neuronal culture. Embryonic DRG neurons were grown in vitro as described previously (Kleitman et al., 1991; Mi et al., 2005). Briefly, the dorsal root ganglia, were dissected from embryonic day 15 Long-Evans rats, plated onto poly-D-lysine coated or collagen coated coverslips for 2 weeks and grown in Neurobasal medium supplemented with B27 (Invitrogen) in the presence of $100 \mathrm{ng} / \mathrm{ml} \mathrm{NGF} \mathrm{(BD} \mathrm{Biosciences,} \mathrm{Franklin}$ Lakes, NJ). DRG cultures were purified by the use of the antimitotic compound, fluorodeoxyuridine $(20 \mu \mathrm{M})$. After 3 weeks in culture, NGF was removed from the cultures for $2 \mathrm{~d}$ before experiments were performed. Purified neurons were infected with indicated lentivirus at 2 multiplicity of infection (MOI) per cell.

OPC/DRG cocultures. A2B5 ${ }^{+}$OPCs and DRG neuronal cultures were prepared as described previously (Mi et al., 2005). One day after OPCs were added to DRG neurons, cocultures were infected with indicated lentivirus at 2 MOI per cell. Cocultures were labeled with antibodies to identify changes in myelination after 2 weeks. Myelin basic protein (MBP) positive oligodendrocytes and myelin internodes were counted. Four-week-old cultures were subjected to SDS-PAGE/Western blot analyses to quantify MBP and myelin-associated glycoprotein (MAG).

Schwann cell/DRG cocultures. Schwann cells were collected from the Long-Evans postnatal day 2 rats as described previously (Chan et al., 2004). Briefly, the sciatic nerves were extracted and incubated in collagenase I for $15 \mathrm{~min}$, and then in $0.25 \%$ trypsin-EDTA (Invitrogen) and collagenase for $15 \mathrm{~min}$ at $37^{\circ} \mathrm{C}$. Cells were plated onto tissue culture plates and grown at $37^{\circ} \mathrm{C}$ in DMEM medium with $10 \%$ fetal calf serum (Invitrogen). Contaminating cell types were eliminated by treating with 5 $\mu \mathrm{M}$ cytosine arabinoside. Schwann cells were seeded onto purified DRGs and infected with lentivirus (2 MOI/cell) $1 \mathrm{~d}$ later.

Lentiviral constructs. DNA sequences used for viral construction are as follows, mouse LINGO-1 full length (FL-LINGO-1; amino acid residues 34-614), LINGO-1 dominant negative (DN-LINGO; amino acid residues $34-581$ ), rat $775^{\text {NTR }}$ full length (FL-p $75^{\text {NTR }}$; amino acid residues $1-425), \mathrm{p} 75^{\mathrm{NTR}}$ dominant negative (DN-p75 ${ }^{\mathrm{NTR}}$; amino acid residues 1-275), human TrkA full length (FL-TrkA; amino acid residues 1-796), TrkA dominant negative (DN-TrkA; amino acid residues 1-441), human Taj full length (FL-Taj; amino acid residues 1-417), Taj dominant negative (DN-Taj; amino acid residues 1-192), rat Nogo receptor full length (FL-NgR; amino acid residues 1-473), and Nogo receptor dominant negative (DN-NgR; amino acid residues $28-310)$. DNA sequences were inserted into the NotI and BamH1 sites of the HRST-IRESeGFP lentiviral vector, as described previously (Mi et al., 2005). Plasmids were transfected into 293FT cells to produce lentivirus as described previously (Rubinson et al., 2003). Oligodendrocytes, Schwann cells, DRG neurons, or cocultures were infected with lentivirus at 2 MOI per cell.

Semiquantitative RT-PCR. Cultures of oligodendrocytes, Schwann cells and DRG neurons were prepared and treated with either $100 \mathrm{ng} / \mathrm{ml}$ of NGF or $100 \mathrm{ng} / \mathrm{ml}$ of brain-derived neurotrophic factor (BDNF) for $24 \mathrm{~h}$ before the mRNA was isolated. Cellular mRNA was extracted by using the Absolutely RNA miniprep kit (Stratagene, La Jolla, CA). Equal amounts of mRNA were subjected to RT-PCR. The primer sets used for PCRs are: LINGO-1 (forward primer 5'-AGAGACATGCGATTGGTGA-3' and reverse primer 5' -AGAGATGTAGACGAGGTCATT3'); TrkA (forward primer 5-TGACTTCGTTGATGCTGGC-3' and reverse primer- $5^{\prime}$-CTGTAGGGCCTGCCCCTCCAC-3').

LINGO-1 transgenic mice. The LINGO-1 transgenic mice were generated using a $4 \mathrm{~Kb}$ DNA fragment expressing the full-length human LINGO-1 under the direction of the human synapsin promoter $(-499$ to +105 ). Founder animals were generated under the B6SJLF2 background and crossed with C57BL/6 mice. LINGO-1 transgenic mice were generated and identified by PCR genotyping of tail biopsies. Primers were constructed spanning a portion of the synapsin promoter and LINGO-1. The primers used for PCR genotyping are sc1-135: 5' primer: CAGTCTGCGGTGGGCAGCGGAGGAGTCGTG and sc1-136: 3' primer: GCCAACCTCCAGTGACTTGAGGTTGTACAG.

Electron microscopy. Spinal cords from mice were isolated, prepared, and sectioned for electron microscopy as described previously (Mi et al., 2005).

\section{Results}

\section{Axonal inhibition of oligodendrocyte differentiation and myelination}

The regulation of axonal myelination by NGF provides a unique and valuable experimental foothold for characterizing the key axonal promoters and inhibitors of myelination. To identify the axonal signals regulated by NGF, we screened various axonal 
signals differentially regulated by NGF that would either promote or inhibit oligodendrocyte differentiation and myelination. These experiments were performed by using the fact that embryonic NGF-dependent DRGs mature to an NGF-independent state in vitro as they do in vivo (Tong et al., 1996, Chan et al., 2004). Recently, LINGO-1 expressed by OPCs was identified as a novel inhibitor of oligodendrocyte differentiation (Mi et al., 2005). Using a function blocking antibody to LINGO-1 (1A7) in the presence of NGF $(100 \mathrm{ng} / \mathrm{ml})$, we observed a significant enhancement in the number of MBP positive oligodendrocytes and myelin internodes when compared with control IgG-treated OPC/DRG cocultures (Fig. 1 A). Similarly, removal of exogenous NGF and depletion with an NGF scavenger (TrkA-Fc), resulted in a similar enhancement of differentiation and myelination by oligodendrocytes (Fig. 1A). These experiments were performed independently in two separate laboratories and were conducted in double blind manner.

In an attempt to investigate the possibility of LINGO-1 as an axonal inhibitor of oligodendrocyte development and myelination, a lentiviral vector encoding the FL-LINGO-1 was transduced into DRG neurons, OPCs, or both cell types in coculture. Expression of the FL-LINGO-1 on either cell type significantly inhibited both oligodendrocyte differentiation and myelination as detected by MBP positive oligodendrocytes and myelin internodes (Fig. $1 B, C$ ). In contrast, disruption of LINGO-1 with the dominant-negative (DN) form on either cell type was sufficient to overcome the inhibitory action and promote differentiation and myelination (Fig. $1 B, C$ ). Because LINGO-1 associates with the Nogo receptor $(\mathrm{NgR})$, TROY/Taj, and p $75^{\mathrm{NTR}}$, the FL- and DNlentiviral constructs were transduced into OPC/DRG cocultures and analyzed for potential effects on the oligodendrocyte differentiation and myelination. As expected, when the OPC/DRG cocultures expressed these constructs, only the DN forms of LINGO-1 and TrkA enhanced the expression of the myelin proteins MBP and MAG (Fig. 1D). These results clearly illustrate that LINGO-1 and TrkA act to specifically inhibit oligodendrocyte differentiation and myelination without any effect from NgR, TROY/Taj, and p $75^{\mathrm{NTR}}$.

To further recapitulate the inhibition of oligodendrocyte myelination by axonal LINGO-1, transgenic mice overexpressing the full-length LINGO-1, under the neuronal promoter synapsin were established
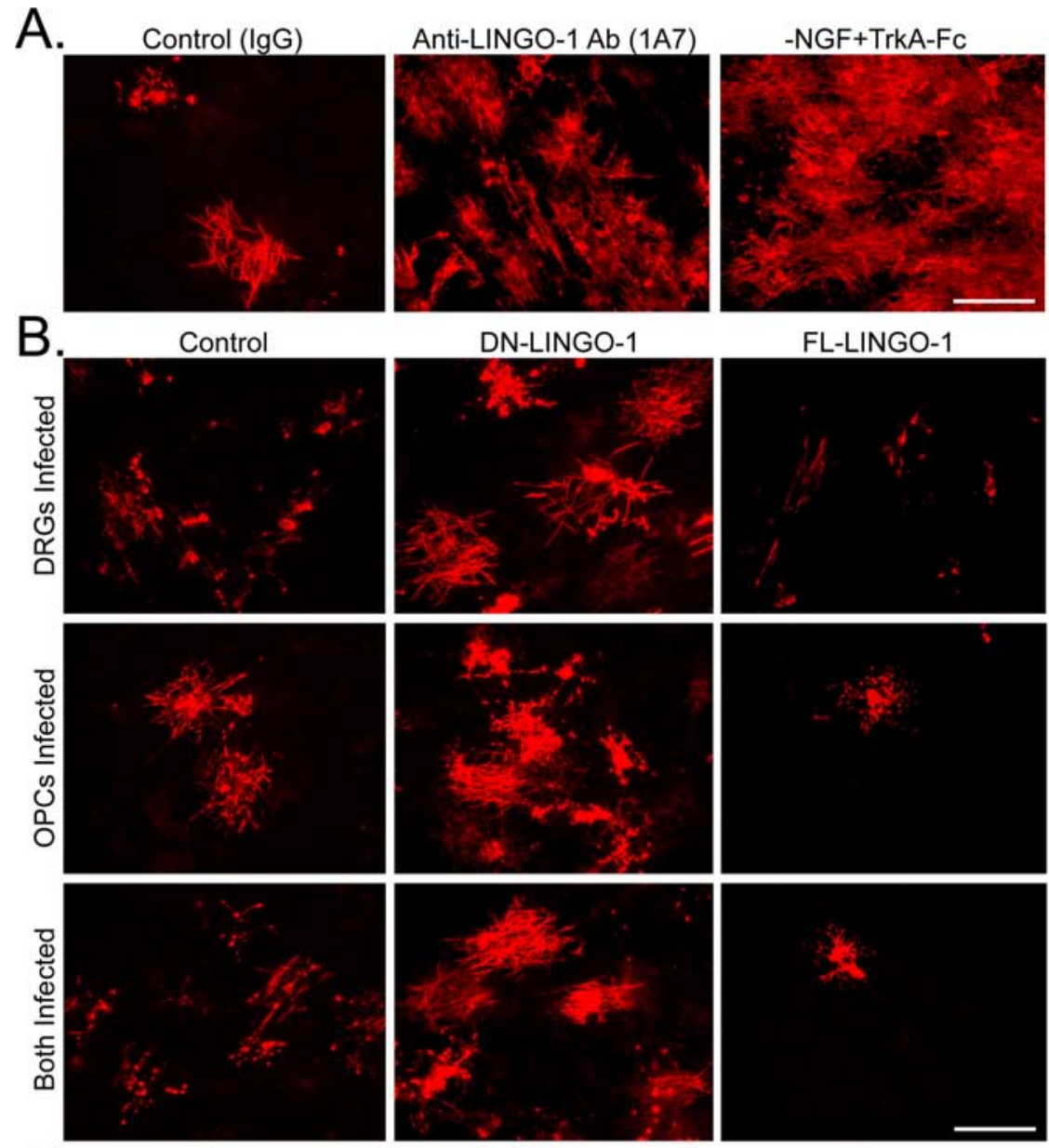

C.

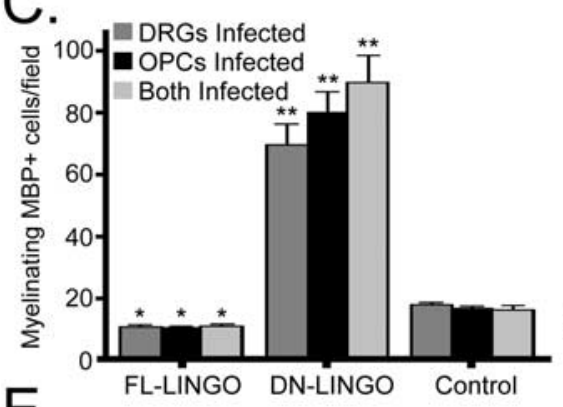

D.

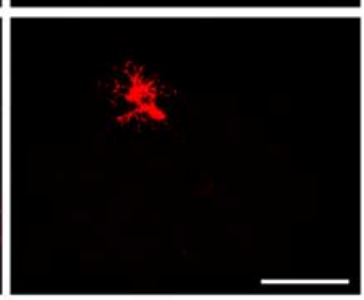

$\mathrm{E}$
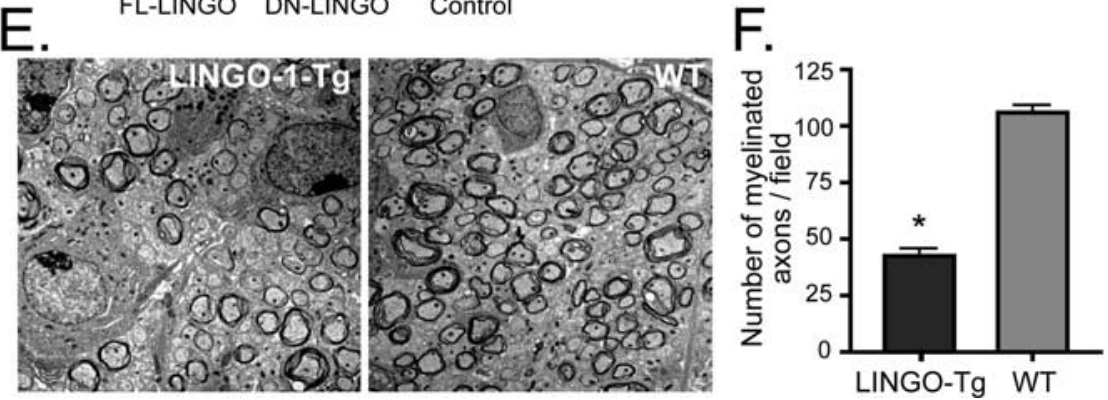

Figure 1. LING0-1 and NGF inhibit oligodendrocyte differentiation and myelination of TrkA positive DRG neurons. $A$, Anti-MBP immunostaining (red) of OPC/DRG cocultures after $14 \mathrm{~d}$ in culture in the presence of a control lgG, an anti-LINGO-1 antibody (1A7) or in the absence of NGF with the addition of the NGF scavenger TrkA-Fc. B, Anti-MBP immunostaining (red) of OPC/DRG cocultures after lentiviral transduction of DN- and FL-LINGO-1 in DRGs, OPCS, or both cell types. $C$, The number of myelinating MBP+ oligodendrocytes/field were quantified for the expression of FL-LINGO-1, DN-LINGO-1, and the control in each of the conditions. ${ }^{*} p<0.05$; ${ }^{* *} p<0.0001$, unpaired $t$ test. $\boldsymbol{D}$, Western blot analysis for MAG, MBP, and $\beta$-actin, of OPC/DRG cocultures infected with FL and DN forms of LING0-1, NgR, TROY/Taj, p75 ${ }^{\mathrm{NTR}}$, and TrkA after $14 \mathrm{~d}$ in culture. $\boldsymbol{E}$, Electron micrographs of spinal cord sections were analyzed for extent of myelination in cross sections from a transgenic mouse overexpressing axonal LING0-1 at postnatal day 8 and a wild-type littermate control. $\boldsymbol{F}$, The number of myelinated axons in the spinal cords were counted and normalized per field. Error bars indicate SD. ${ }^{*} p<0.001$, unpaired $t$ test. Scale bars, $100 \mu \mathrm{m}$. 


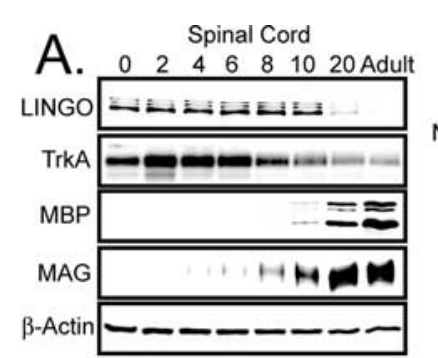

E.

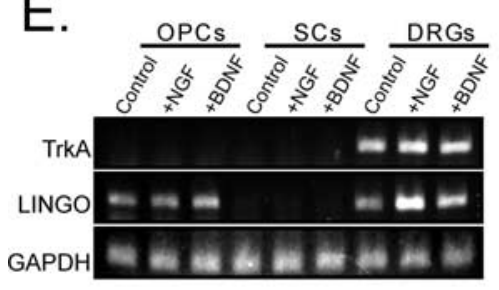

B.

OPC/DRG Cocultures

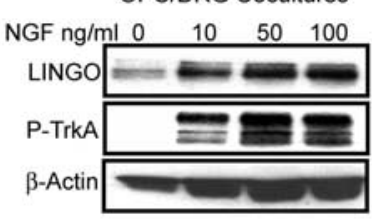

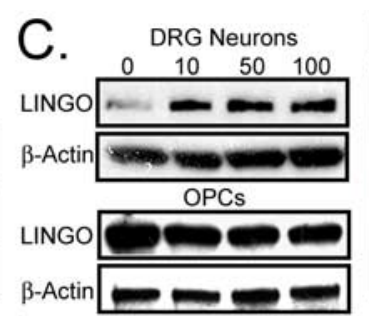
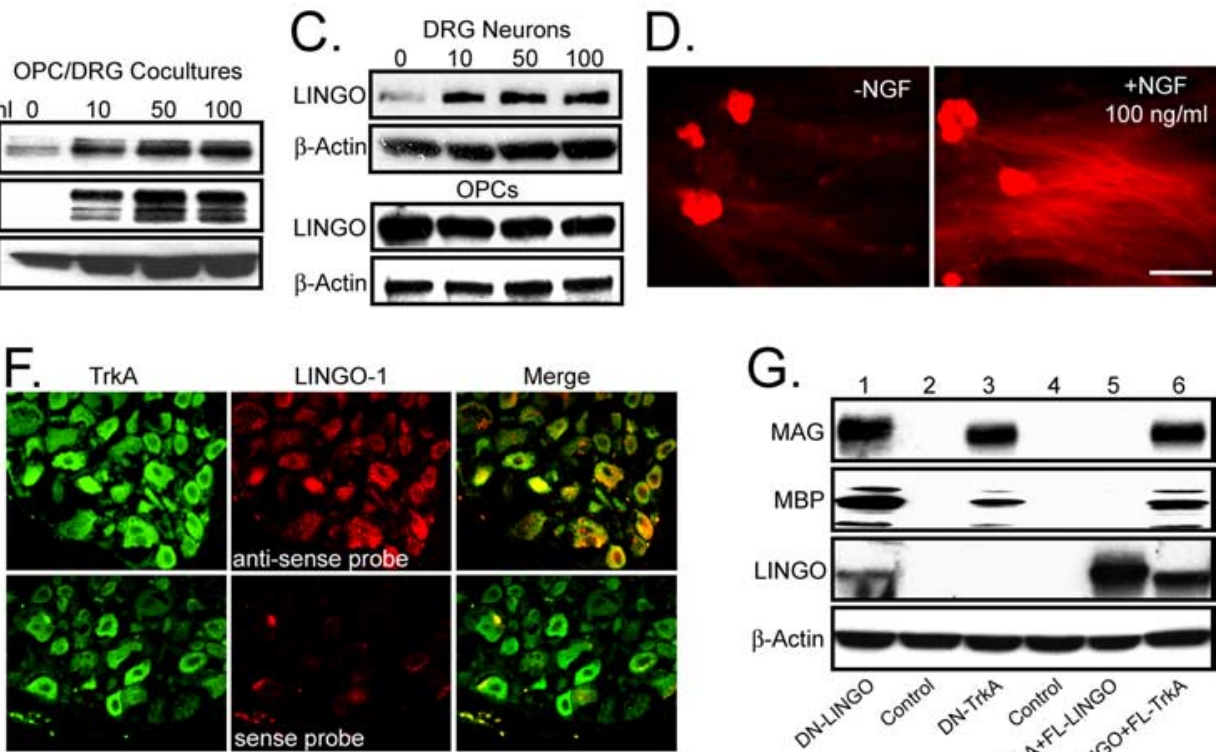

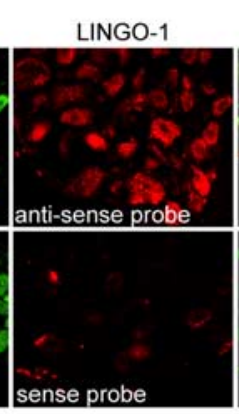

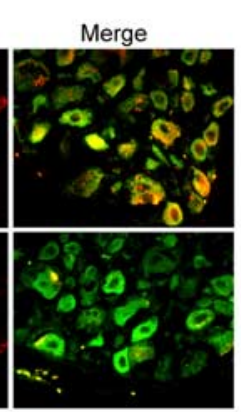

G.

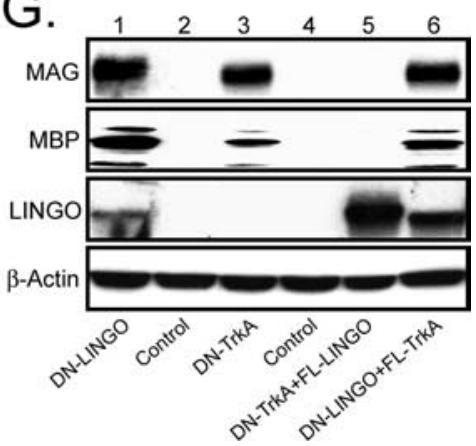

Figure 2. NGF/TrkA regulates the axonal expression of LING0-1 on NGF-dependent DRG neurons. $A$, Expression of LING0-1, TrkA, MBP, MAG, and $\beta$-actin were examined in the developing mouse spinal cord. Tissue from postnatal day $0,2,4,6,8,10,20$, and adult were isolated and prepared for Western blot analysis. B, OPC/DRG cocultures were subjected to NGF treatment for $2 \mathrm{~d}(0-100$ $\mathrm{ng} / \mathrm{ml}$ ) and immunoprecipitated for TrkA. Samples were probed for phosphorylated (P)-tyrosine, LING0-1, and $\beta$-actin. C, Purified DRGs and OPCs were subjected to NGF treatment (0-100 ng/ml) in similar manner, and cells were extracted and prepared for Western blot analysis for LINGO-1 and $\beta$-actin. D, Purified DRG neuronal cultures were either treated with or without NGF (100 ng/ml) and immunostained for LINGO-1. Scale bar, $100 \mu \mathrm{m}$. E, RT-PCR TrkA, LINGO-1, and glyceraldehyde phosphate dyhydrogenase (GAPDH; control) was performed on purified OPCS, SCS, and DRGs in the presence or absence of NGF or BDNF. F, Combined immunostaining for TrkA and in situ hybridization for LING0-1 mRNA were performed on frozen sections of rat postnatal day 6 dorsal root ganglia. Sections were cut and probed with a digoxigenin-labeled RNA for LING0-1 (red) and then with an antibody to TrkA (green). A sense probe for LING0-1 was used as a control for the in situ hybridization. G, LING0-1 inhibits oligodendrocyte differentiation and myelination downstream of NGF/TrkA signaling. DRG cultures were infected for $2 \mathrm{~d}$ with DN-LING0-1 (1), control (2), DN-TrkA (3), control (4), or a combination of DN-TrkA and FL-LING0-1 (5) or DN-LING0-1 and FL-TrkA (6). Purified OPCs were seeded onto the DRG cultures after infection and allowed to grow for 14 d. Cocultures were then extracted and prepared for Western blot analysis. Samples were probed for MAG, MBP, LING0-1, and $\beta$-actin.

(Fig. $1 E$, supplemental Fig. $1 A, B$, available at www.jneurosci.org as supplemental material). The transgenic mice were confirmed as described in the Material and Methods section. The spinal cords of transgenic mice with enforced axonal LINGO-1 displayed a significant reduction in the number of myelinated axons at $8 \mathrm{~d}$ postnatal as compared with wild-type spinal cord controls (Fig. $1 E, F)$. This reduction in myelination was also observed in the brain of the transgenic mice (data not shown). Although myelination in the transgenic mice seems to recover at later stages, the myelin extent was never completely restored. Together, the in vitro and in vivo data suggest that LINGO-1 is a potent axonal inhibitor of oligodendrocyte differentiation and myelination.

\section{NGF regulates axonal expression of LINGO-1 to inhibit myelination of TrkA-positive DRGs}

Initially, we set out to determine the expression of LINGO-1 and TrkA in the developing wild-type spinal cord during development. We found a striking correlation in the expression of LINGO-1 and TrkA, and the induction of the myelin proteins MBP and MAG after the downregulation of LINGO-1 and TrkA (Fig. 2A). To determine whether LINGO-1 expression may be regulated by NGF and its receptor TrkA, OPC/DRG cocultures were treated NGF $(0-100 \mathrm{ng} / \mathrm{ml})$ for $2 \mathrm{~d}$ and analyzed for LINGO-1 expression and the corresponding activation of TrkA by immunostaining and Western blot. The cocultures were extracted after NGF treatment and immunoblotted for LINGO-1 or immunoprecipitated with a Trk antibody and then immunoblotted for phosphorylated tyrosine. The addition of NGF led to the activation of TrkA and an induction in LINGO-1 expression (Fig.
$2 B)$. We next treated purified DRGs and OPCs separately with NGF in a dose-dependent manner $(0-100 \mathrm{ng} / \mathrm{ml})$. The cultures were then assayed for LINGO-1 expression by Western analysis (Fig. 2C). LINGO-1 expression was induced by NGF in DRG neurons but not in the OPC cultures. To further confirm this study, LINGO-1 expression was analyzed by immunostaining purified DRGs in the presence or absence of NGF. LINGO-1 was localized to the neuronal cell bodies and to the axons of the DRGs, with the latter being dramatically upregulated by NGF (Fig. 2D). Two approaches were used to determine the expression and localization of LINGO-1 and TrkA. First, we examined the expression of both TrkA and LINGO-1 in OPCs, SCs, and DRGs by semiquantitative RT-PCR (Fig. 2E). Although OPCs expressed LINGO-1, they did not express TrkA. SCs did not express detectable levels of mRNA for either molecule, whereas DRGs expressed both TrkA and LINGO-1. By treating with NGF or the neurotrophin BDNF, it was evident that NGF specifically induced LINGO-1 transcription in the DRGs. Secondly, to determine the localization of LINGO- 1 and TrkA in vivo, we examined the coexpression of TrkA and LINGO-1 in the dorsal root ganglia from postnatal rat pups by TrkA immunostaining followed by in situ hybridization for LINGO-1. TrkA positive DRGs clearly expressed mRNA for LINGO-1 (Fig. 2F). A sense probe was used as a control for the in situ hybridization.

To determine whether the expression and inhibitory effects of LINGO-1 are downstream of TrkA activation, we infected purified DRGs in the presence of NGF $(100 \mathrm{ng} / \mathrm{ml})$ with lentiviruses transducing combinations of DN and FL forms of LINGO-1 and TrkA. Purified OPCs were then seeded onto the DRGs after infection. Consistent with the previous findings (Chan et al., 2004; 

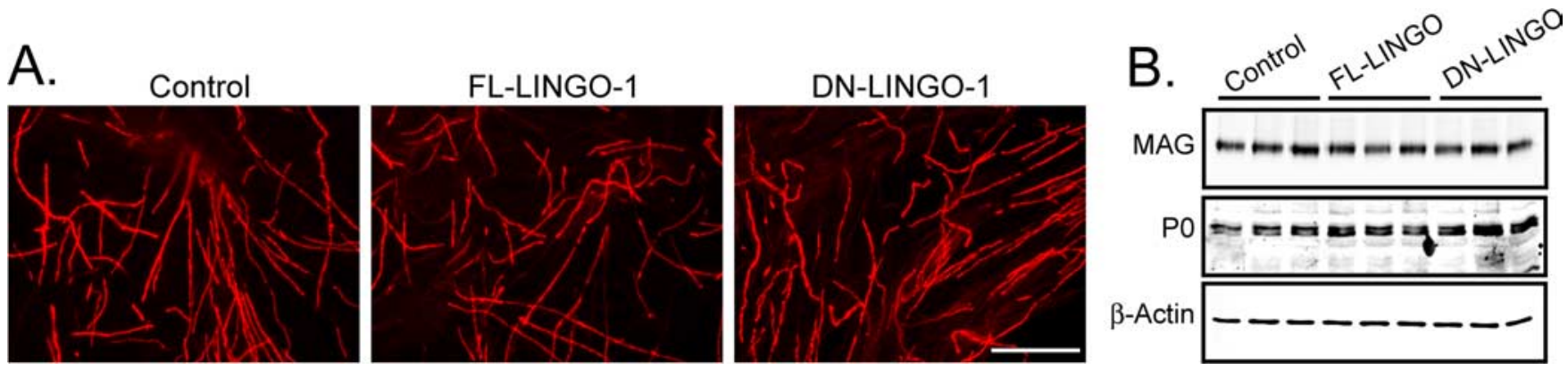

Figure 3. LING0-1 does not influence Schwann cell myelination. $\boldsymbol{A}, \boldsymbol{B}$, Lentivirus was used to transduce FL- and DN-LING0-1 in the SC/DRG cocultures and myelination was analyzed by immunostaining for MBP and Western blot analysis for MAG, $\mathrm{PO}$, and $\beta$-actin. The expression of the myelin proteins and the formation of myelin internodes were unaltered by the expression of the FL- or DN-LING0-1. Scale bar, $100 \mu \mathrm{m}$.

Mi et al., 2005), expression of FL-LINGO-1 or FL-TrkA acts to inhibit oligodendrocyte differentiation and myelination, whereas expression of DN-LINGO-1 or DN-TrkA significantly enhances oligodendrocyte differentiation and myelination in the presence of NGF (Figs. $1 D, 2 G$ ). However, in the presence of FL-LINGO-1, DN-TrkA is not sufficient to induce differentiation and myelination of oligodendrocytes, whereas DN-LINGO-1 is sufficient to elicit differentiation of oligodendrocytes in the presence of FLTrkA (Fig. 2G). Together, our results suggest that the effects of LINGO-1 are downstream of TrkA activation.

\section{Axonal LINGO-1 does not influence Schwann cell myelination}

When examining the developmental profile for the expression of LINGO-1 in the PNS (sciatic nerve) (data not shown) and CNS (spinal cord), it became quite evident that in the spinal cord exclusively, the downregulation of LINGO-1 correlated with a decrease in TrkA expression and the initiation of myelination (MBP and MAG expression) (Fig. 2A). These results seem to confirm our findings that LINGO-1 is regulated by NGF and TrkA and that LINGO-1 is a potent inhibitor of OPC differentiation and myelination.

Whereas NGF/TrkA inhibits myelination by oligodendrocytes, it promotes myelination by SCs (Chan et al., 2004). These findings provide direct evidence that the axonal signals regulating myelination differ between the central and peripheral nervous systems, based on the fact that NGF could not simultaneously activate and inhibit the same axonal signal. With this in mind, we set out to determine whether axonal LINGO-1 has any effect on SC myelination. Using SC/DRG cocultures, established as described previously (Chan et al., 2004), the forced expression of FL- and DN-LINGO-1 in SC/DRG cocultures was used to determine the role, if any, of LINGO-1 on SC myelination. Myelination by SCs was analyzed by immunostaining for MBP and Western blot analysis for MAG and protein zero (P0) (Fig. $3 A, B$ ). The expression of the myelin proteins and the formation of myelin internodes were unaltered by the expression of the FL- or DNLINGO-1, indicating that LINGO-1 does not influence Schwann cell myelination. Additionally, we did not detect any significant change in the number of myelinated fibers in the peripheral nervous system, consistent with our in vitro findings (data not shown).

\section{Discussion}

It is generally thought that during development axons control whether they will become myelinated by expressing appropriate signals to either promote or inhibit this process. Because some axons are myelinated and others remain unmyelinated, what are the factors that determine the expression of these signals along axons and how are these signals regulated throughout development? Our recent finding that NGF controls myelination of TrkA-expressing neurons represents an ideal model for detailed examination of the axonal induction of myelination. Identification of the axonal factor(s) regulated by NGF represents a pivotal step in developing a greater understanding of myelination by oligodendrocytes. Our results indicate that LINGO-1 is a potent axonal inhibitor of oligodendrocyte differentiation and myelination, regulated by NGF and its receptor TrkA. Whereas LINGO-1 expressed by OPCs was previously identified as an inhibitor of differentiation and myelination (Mi et al., 2005), we demonstrate that axonal expression of LINGO-1 inhibits differentiation and myelination with equal potency. Disruption of LINGO-1 on either cell type is sufficient to overcome the inhibitory action and promote differentiation and myelination of oligodendrocytes independent of axon caliber. Whereas it has long been thought that the induction of myelination is intimately related to axon diameter (Voyvodic, 1989; Colello and Pott, 1997), the effect of NGF/ TrkA/LINGO-1 represents a unique instance in which NGF promotes an increase in axon diameter, which results in an inhibition in oligodendrocyte myelination (Markus et al., 2002). These findings provide us with a unique model system in which we can now investigate the role of axonal factors in myelination, independent of the effects of axon diameter

The regulation of axonal myelination signals by NGF seems to be consistent with an emerging model in which the growth factors that promote the early survival and differentiation of particular sets of neurons, later play an important role in regulating the axonal signals that control whether those axons will become myelinated. Recent evidence indicates that overexposure to GDNF, a growth factor important in the development of a subset of nociceptive neurons, can increase the proportion of those axons that are myelinated in the peripheral nervous system, although direct effects of GDNF on Schwann cells cannot be excluded (Hoke et al., 2003). Moreover, hypomyelination of the optic nerve is observed in mice lacking BDNF, a growth factor important in the development of optic axons but not known to influence the development of optic nerve oligodendrocytes (Cellerino et al., 1997). An interesting question is whether this phenomenon of extrinsic regulation of axonal myelination signals, such as LINGO-1, by growth factors extends to all neurons or whether it is restricted to certain classes of neurons (i.e., the nociceptive neurons) that ultimately need to decide to either become myelinated or remain unmyelinated. The implications of these findings 
relate specifically to the identification of new target molecules and extrinsic growth factors necessary for establishing the appropriate environment for myelination. It is our hope that this study may provide a new therapeutic target for the treatment of demyelination and dysmyelination disorders such as Multiple Sclerosis.

\section{References}

Cellerino A, Carroll P, Thoenen H, Barde YA (1997) Reduced size of retinal ganglion cell axons and hypomyelination in mice lacking brain-derived neurotrophic factor. Mol Cell Neurosci 9:397-408.

Chan JR, Watkins TA, Cosgaya JM, Zhang C, Chen L, Reichardt LF, Shooter EM, Barres BA (2004) NGF controls axonal receptivity to myelination by Schwann cells or oligodendrocytes. Neuron 43:183-191.

Colello RJ, Pott U (1997) Signals that initiate myelination in the developing mammalian nervous system. Mol Neurobiol 15:83-100.

Hoke A, Ho T, Crawford TO, LeBel C, Hilt D, Griffin JW (2003) Glial cell line-derived neurotrophic factor alters axon schwann cell units and promotes myelination in unmyelinated nerve fibers. J Neurosci 23:561-567.

Kleitman N, Wood PM, Bunge RP (1991) Tissue culture methods for the study of myelination. In: Culturing nerve cells (Banker G, Goslin K, eds), pp 337-377. Cambridge, MA: MIT.

Markus A, Zhong J, Snider WD (2002) Raf and akt mediate distinct aspects of sensory axon growth. Neuron 35:65-76.
Mi S, Lee X, Li X, Veldman GM, Finnerty H, Racie L, LaVallie E, Tang XY, Edouard P, Howes S, Keith JC, McCoy JM (2000) Syncytin is a captive retroviral envelope protein involved in human placental morphogenesis. Nature 403:785-789.

Mi S, Miller RH, Lee X, Scott ML, Shulag-Morskaya S, Shao Z, Chang J, Thill G, Levesque M, Zhang M, Hession C, Sah D, Trapp B, He Z, Jung V, McCoy JM, Pepinsky RB (2005) LINGO-1 negatively regulates myelination by oligodendrocytes. Nat Neurosci 8:745-751.

Michailov GV, Sereda MW, Brinkmann BG, Fischer TM, Haug B, Birchmeier C, Role L, Lai C, Schwab MH, Nave KA (2004) Axonal neuregulin-1 regulates myelin sheath thickness. Science 304:700-703.

Rubinson DA, Dillon CP, Kwiatkowski AV, Sievers C, Yang L, Kopinja J, Rooney DL, Ihrig MM, McManus MT, Gertler FB, Scott ML, Van Parijs L (2003) A lentivirus-based system to functionally silence genes in primary mammalian cells, stem cells and transgenic mice by RNA interference. Nat Genet 33:401-406.

Taveggia C, Zanazzi G, Petrylak A, Yano H, Rosenbluth J, Einheber S, Xu X, Esper RM, Loeb JA, Shrager P, Chao MV, Falls DL, Role L, Salzer JL (2005) Neuregulin-1 type III determines the ensheathment fate of axons. Neuron 47:681-694.

Tong JX, Eichler ME, Rich KM (1996) Intracellular calcium levels influence apoptosis in mature sensory neurons after trophic factor deprivation. Exp Neurol 138:45-52.

Voyvodic JT (1989) Target size regulates calibre and myelination of sympathetic axons. Nature 342:430-433. 